\title{
Dissociable Roles for Cortical and Subcortical Structures in Memory Retrieval and Acquisition
}

\author{
Anna S. Mitchell, Philip G. F. Browning, Charles R. E. Wilson, Mark G. Baxter, and David Gaffan \\ Department of Experimental Psychology, Oxford University, Oxford OX1 3UD, United Kingdom
}

The relationship between anterograde and retrograde amnesia remains unclear. Previous data from both clinical neuropsychology and monkey lesion studies suggest that damage to discrete subcortical structures leads to a relatively greater degree of anterograde than retrograde amnesia, whereas damage to discrete regions of cortex leads to the opposite pattern of impairments. Nevertheless, damage to the medial diencephalon in humans is associated with both retrograde and anterograde amnesia. In the present study, we sought to reconcile this by assessing retention as well as subsequent relearning and new postoperative learning. Rhesus monkeys learned 300 unique scene discriminations preoperatively, and retention was assessed in a preoperative and postoperative one-trial retrieval test. Combined bilateral subcortical lesions to the magnocellular mediodorsal thalamus and fornix impaired postoperative retention of the preoperatively acquired information. In addition, subsequent relearning and new postoperative learning were also impaired. This contrasts with the effects of a discrete lesion to just one of these structures, after which retention is intact in both cases. Discrete bilateral ablations to the entorhinal cortex impaired retention but had no effect on new learning. Combined with previous work from our laboratory, these results support the hypothesis that subcortical damage has a relatively greater effect on new learning, and cortical damage has a relatively greater effect on retention. Furthermore, the results demonstrate that retrograde amnesia occurs as a result of subcortical damage only if it is widespread, leading to an extensive disruption of cortical functioning. Damage of this nature may account for dense amnesia.

Key words: mediodorsal thalamus; fornix; entorhinal cortex; amnesia; acquisition; retrieval

\section{Introduction}

Although damage to the medial diencephalon in the human brain is associated with both retrograde and anterograde amnesia (Graff-Radford et al., 1990; Hodges and McCarthy, 1993; Kopelman et al., 1999; Kopelman, 2002; Miller et al., 2003; Van der Werf et al., 2003), there is some evidence to indicate that the processes underlying these deficits may be independent of each other. Rhesus monkeys with neurotoxic lesions of the magnocellular mediodorsal thalamus (MDmc) unexpectedly show no impairment in retention of preoperatively acquired scene discriminations, in contrast to a substantial impairment in new postoperative learning of novel scene discriminations (Mitchell and Gaffan, 2008). This study indicates that on its own, MDmc is necessary for encoding of new information but not for retrieval of previously acquired information. Similar results demonstrating substantial deficits in new learning with minimal impairments in retention have been reported after damage to the fornix (Fx) (Gaffan, 1993a; Buckley et al., 2008). The Fx links several structures involved in processing episodic memories, including the

Received March 14, 2008; revised June 11, 2008; accepted June 23, 2008.

This work was supported by the Medical Research Council (UK). We thank M. Brown, P. Croxson, G. Daubney, K. Murphy, and S. Mygdal for technical support.

Correspondence should be addressed to Dr. Anna S. Mitchell, Department of Experimental Psychology, Oxford University, South Parks Road, 0xford OX1 3UD, UK. E-mail: anna.mitchell@psy.ox.ac.uk.

DOI:10.1523/JNEUROSCI.1924-08.2008

Copyright $\odot 2008$ Society for Neuroscience $\quad$ 0270-6474/08/288387-10\$15.00/0 mammillary bodies, anterior thalamus, and extended hippocampal system (Saunders and Aggleton, 2007).

Subcortical damage leading to amnesia in humans is not restricted to a single structure. We propose that dense amnesia may be more readily explained if the contributions of various subcortical structures and/or pathways are assessed together, and this can be tested by assessing the effects of combined subcortical lesions. We predict that subcortical damage will lead to widespread cortical dysfunction, and consequently dense amnesia, only when it affects multiple neuromodulatory influences (Aggleton and Brown, 2006).

The effects of subcortical lesions were contrasted with those of selective cortical lesions, because widespread damage limited to cortex may cause relatively greater deficits in retention of preoperatively acquired information than new learning (Thornton et al., 1997; Bright et al., 2006; Browning and Gaffan, 2008). We considered the entorhinal cortex (ERh), because it is unambiguously part of the putative medial temporal lobe memory system (Squire and Zola-Morgan, 1991), yet its involvement in retention and new learning remains unclear, largely because studies in monkeys typically also damage the perirhinal cortex (Leonard et al., 1995; Meunier et al., 1996; Charles et al., 2004).

We report here the effects of subcortical neurotoxic lesions to MDmc combined with fornix transection $(\mathrm{MD}+\mathrm{Fx})$, and also selective cortical ablations of ERh, on retention of 300 unique scene discriminations learned preoperatively using a preoperative and postoperative one-trial retrieval test (experiment 1), re- 
learning of these 300 discriminations (experiment 2), and new postoperative learning of 100 novel scene discriminations (experiment 3). If widespread damage to subcortical neural systems results in widely disrupted cortical function, then combined damage to $\mathrm{MD}+\mathrm{Fx}$ should result in retrograde amnesia as well as severe impairments in new learning, although damage to either structure alone is without effect on retention of preoperatively learned information. In contrast, if cortical structures are preferentially involved in retention of preoperatively acquired information, then limited cortical damage (in this case to ERh) should produce retrograde but not anterograde amnesia, a double dissociation to the effects of lesions of single subcortical systems.

\section{Materials and Methods}

Animals

Eleven male naive rhesus monkeys (Macaca mulatta, $3.08-8.60 \mathrm{~kg}$, between 2.5 and 5 years old), at the beginning of behavioral training, participated in this study. Three monkeys (MD+Fx1, MD+Fx2, and $\mathrm{MD}+\mathrm{Fx} 3$ ) formed the combined lesion group involving neurotoxic bilateral lesions to the magnocellular division of the mediodorsal thalamic and a bilateral fornix transection $(\mathrm{MD}+\mathrm{Fx})$. Three monkeys (ERh1, ERh2, and ERh3) formed the entorhinal lesion group involving selective bilateral ablations to entorhinal cortex. The remaining five monkeys (CON1, CON2, CON3, CON4, and CON5) were unoperated controls. Each monkey was preoperatively trained to a stable level of performance on the same 300 unique object-in-place scene discriminations before surgery. All experimental procedures were performed in compliance with the United Kingdom Animals Scientific Procedures Act (1986).

\section{Apparatus}

The computer-controlled test apparatus was identical to that described in detail previously by Mitchell et al. (2007b). Briefly, it consisted of a large touch-sensitive color monitor that displayed the visual stimuli and food-reward dispensers. A food-reward cup was positioned directly under the touch screen, where $190 \mathrm{mg}$ banana-flavored pellets (P. J. Noyes) were dispensed for each correct response, and a metal "lunch box" was located to the left of the food-reward cup (filled with a mixture of wet monkey chow, seeds, apple, banana, orange, nuts, and dates), which was automatically opened after the last correct trial per testing session was completed.

\section{Scene-discrimination learning}

The scene-discrimination learning task was adapted from Gaffan (1994). The stimulus material was identical to those described in detail along with example stimuli (Mitchell et al., 2007b). Briefly, each trial consisted of an artificially constructed "scene." There were two foreground objects in each scene, one correct (rewarded) and the other incorrect (nonrewarded), consisting of randomly selected, small, colored typographic characters each placed in a constant location. Each scene was unique in that it varied in several randomly selected attributes, including (1) the background color of the screen; (2) the location of ellipses on the screen; (3) the color, size, and orientation of ellipse segments; (4) the typographic character, clearly distinct in size from the foreground objects; and (5) the color of the typographic character. All the colors were assigned with the constraint that the foreground objects should be visible (i.e., there was a minimum separation in color space between the colors of a foreground object and the color of any element of its local background).

\section{Behavioral training}

A detailed explanation of pretraining was provided by Mitchell et al. (2007b). When the monkeys were reliably touching the foreground object when presented with a new scene and completing 60 trials per day with minimal accuracy errors (i.e., touching any location on the screen other than the foreground typographic characters), behavioral training began in initial introductory stages. For the first stage, all of the monkeys were presented with 25 scene discriminations, and they had to learn these scenes for a minimum of 10 sessions; a session involved the completion of all 25 scene discriminations. They were then presented with 50 scene discriminations and again had to learn these scenes for a minimum of 10 sessions. Finally, all monkeys were presented with 100 scene discriminations, and from this point onward in the experiment, a session consisted of the completion of 100 scene discriminations. Monkeys had to learn these 100 scenes until they performed at $90 \%$ correct or better across two consecutive sessions. At this point, training for the 300 scene discriminations (divided into three sets of 100 discriminations each) began. All monkeys learned the first set of 100 scenes (set A) to a stable criterion set at $90 \%$ or above across two consecutive sessions. On reaching this criterion, each monkey learned the second set of 100 scenes (set B) until performance was at $90 \%$ correct or above across two consecutive sessions, and then they learned the third set of 100 scenes (set C) until $90 \%$ correct or above across two consecutive sessions. During the scenediscrimination training, each scene was presented with two foreground objects (one correct and one incorrect, as described above). A touch to the correct object caused the object to flash for $2 \mathrm{~s}$, and then the screen blanked and a reward pellet was delivered into the hopper. A touch to the incorrect object caused the screen to blank immediately; no reward was given, and an intertrial interval was imposed for $10 \mathrm{~s}$. A correction trial was then administered in which the scene was re-presented with only the correct object present. Touches anywhere else in the scene caused the screen to blank and the trial was repeated. When the monkey completed the final trial of a session, the lunch box opened automatically, and the monkey received the large food reward and was allowed time to eat the food before being returned to the home enclosure.

\section{Experiment 1: one-trial preoperative and postoperative} retrieval tests

After reaching the behavioral performance criterion on the third set (set $\mathrm{C}$ ), the preoperative retrieval test was conducted on the following day. The first day consisted of "familiarization"; the monkeys saw 100 novel scenes using the scene-discrimination task, but with only one random typographic character presented against the standard scene background (these trials being identical to correction trials). The monkeys had to touch the single typographic character to receive a reward. Responses to anywhere else on the screen immediately ended the trial, and it was re-presented after a $10 \mathrm{~s}$ delay. This familiarization ensured that the monkeys had not altered their motivation as a consequence of the extended break from testing that they would experience as a consequence of "postoperative recovery." On the second day, the monkeys were presented with set A, using the same testing methods experienced during preoperative training. On the third day, set $\mathrm{B}$ was presented, and on the fourth day, set $\mathrm{C}$ was presented. These three final consecutive days of testing (days 2-4) constituted the one-trial preoperative retrieval test. Monkeys were then matched based on their performance and assigned to the MD+Fx lesion group, the ERh lesion group, or the unoperated control group. MD+Fx and ERh monkeys were scheduled for surgery, and the others were scheduled for an equivalent period of rest. After postoperative recovery of at least $12 \mathrm{~d}$, all monkeys were tested on the one-trial postoperative retrieval test, which was identical to the preoperative retrieval test.

\section{Experiment 2: relearning of the 300 scene discriminations}

After the postoperative retrieval test, each monkey continued testing for three further cycles of the retrieval test described above, using only sets A, $\mathrm{B}$, and $\mathrm{C}$. This testing period did not include any further sessions using familiarization. This experiment was designed to test the relearning of the preoperatively acquired material.

\section{Experiment 3: acquisition of 100 novel scene discriminations}

After the three further cycles of relearning sets A, B and C, each monkey learned one additional set of 100 unique and novel scene discriminations (set D). The type of stimuli and rewards used in this task were identical to the retention memory task. Correction trials, as described above, were used when an error occurred. The dependent measure was the number of errors made to reach a behavioral criterion of at least $90 \%$ correct in two consecutive sessions.

\section{Surgical procedures}

A more detailed description of the surgical methods and preoperative and postoperative drug treatments was provided by Mitchell et al. 
(2007a). Briefly, each monkey was sedated on the morning of surgery with both ketamine and xylazine and then intubated and, during surgery, was mechanically ventilated and maintained deeply anesthetized using both gaseous [sevoflurane (1.4-3.0\%, to effect, in 100\% oxygen)] and intravenous [alfentanil $(0.5-3.0 \mu \mathrm{g} / \mathrm{kg})$ ] anesthesia.

For MD+Fx lesions, three monkeys received the bilateral neurotoxic lesions to the magnocellular division of the mediodorsal thalamic nucleus, combined with bilateral fornix transection in one neurosurgical procedure. In MD1, the MDmc lesion was completed first, followed by fornix transection, whereas in MD2 and MD3, the Fx transection was completed first, followed by the MDmc lesion. Each monkey was placed in a stereotaxic head holder, and after the skin and underlying galea were opened, and a right-sided D-shaped bone flap was created, the dura over the posterior part of the right hemisphere was cut and retracted to the midline. The splenium of the corpus callosum was cut in the midline with a glass aspirator. The tela choroidea was cauterized in the midline posterior and dorsal to the thalamus, using a metal aspirator that was insulated to the tip. A stereotaxic manipulator holding a $10 \mu \mathrm{l}$ Hamilton syringe with a blunt-tipped 26 gauge needle was positioned above the posterior commissure at the midline, using the third ventricle as a guide. Neurotoxic bilateral lesions to the MDmc were produced by $10 \times 1 \mu \mathrm{l}$ of injections of a combined mixture of ibotenic acid $(10 \mathrm{mg} / \mathrm{ml}$; Biosearch Technologies) and NMDA (10 mg/ml; Tocris Bioscience) dissolved in sterile $0.1 \mathrm{~m}$ PBS. The monkey brain atlas of Ilinsky and Kultas-Ilinsky (1987) was used to calculate the intended lesion site coordinates. The needle was positioned for the first pair of coordinates: anteroposterior (AP), $+5.2 \mathrm{~mm}$ anterior to the posterior commissure; mediolateral (ML), $\pm 1.2 \mathrm{~mm}$ lateral to the third ventricle; and dorsoventral (DV), $-4.0 \mathrm{~mm}$ (to compensate for the hole positioned $1 \mathrm{~mm}$ above the tip of the needle) ventral to the surface of the thalamus directly above the intended lesion site. Each injection was made slowly over $4 \mathrm{~min}$, and the needle was left in place for $\sim 4$ min before being moved to the next site. The needle was then repositioned for the second pair of coordinates: AP, $+4.2 \mathrm{~mm}$; ML, $\pm 1.5 \mathrm{~mm}$; and DV,$-5.0 \mathrm{~mm}$. The third, fourth, and fifth pairs of coordinates were $\mathrm{AP},+4.2 \mathrm{~mm}, \mathrm{ML}, \pm 1.5 \mathrm{~mm}$, and $\mathrm{DV},-3.0$ $\mathrm{mm}$; AP, $+3.4 \mathrm{~mm}, \mathrm{ML}, \pm 1.7 \mathrm{~mm}$, and $\mathrm{DV},-4.0 \mathrm{~mm}$; and $\mathrm{AP},+3.4$ $\mathrm{mm}, \mathrm{ML}, \pm 1.7 \mathrm{~mm}$, and $\mathrm{DV},-3.0 \mathrm{~mm}$, respectively. In each case, the DV coordinate reading was taken from the surface of the thalamus directly above the intended injection site.

For the fornix transection, the left hemisphere was retracted from the falx with a brain spoon. A glass aspirator was used to make a sagittal incision no more than $5 \mathrm{~mm}$ in length in the corpus callosum at the level of the interventricular foramen. The fornix was sectioned transversely by electrocautery and aspirated with a 20 gauge metal aspirator that was insulated to the tip. When the combined lesions were complete, the dura was repositioned but not sewn, the bone flap was replaced and held with sutures, and the skin and galea were closed in layers. Nonsteroidal antiinflammatory analgesic (meloxicam, $0.2 \mathrm{mg} / \mathrm{kg}$, oral) and antibiotic $(8.75 \mathrm{mg} / \mathrm{kg}$, oral $)$ treatment continued after surgery in consultation with veterinary staff, typically for $5 \mathrm{~d}$.

For entorhinal ablations, three monkeys received the bilateral ablations to the entorhinal in one neurosurgical procedure, using only a subfrontal approach. The arch of the zygoma was removed, and the temporal muscle was detached from the cranium and retracted. A bone flap was raised over the frontal and temporal lobe. The medial and posterior limits of the bone flap were in a crescent shape extending from within $5 \mathrm{~mm}$ of the midline at the brow to the posterior insertion of the zygomatic arch. The anterior limit of the flap was the brow and the orbit. Ventrally, the flap extended from the posterior insertion of the zygomatic arch to the level of the superior temporal sulcus in the lateral wall of the temporal fossa anteriorly. The ventral anterior part of this bone opening was then extended with a rongeur ventrally into the wall of the temporal fossa to reach the base of the temporal fossa. The dura mater was incised to expose the dorsolateral frontal and lateral temporal lobe. The frontal lobe was retracted from the orbit with a brain spoon to enable access to the anterior medial temporal lobe. Pia mater was cauterized, and the underlying cortex was removed by aspiration in the medial bank of the anterior part of the rhinal sulcus; and in the medially adjacent $2 \mathrm{~mm}$ of cortex, the removal was extended as far posteriorly as possible toward the
Table 1. Percentage of damage to area MDmc in six monkeys with MDmc lesions

\begin{tabular}{ll}
\hline Monkey & Damage to MDmc \\
\hline MD+Fx1 & 92.50 \\
MD+Fx2 & 94.47 \\
MD+Fx3 & 99.09 \\
Mean & 95.35 \\
MD4 & 81.71 \\
MD5 & 78.56 \\
MD6 & 95.02 \\
Mean & 85.09 \\
\hline
\end{tabular}

$M D+F \times 1, M D+F \times 2$, and $M D+F \times 3$ are the three monkeys from the current study with combined bilateral fornix transection; MD4, MD5, and MD6 are three monkeys from a previously published study (Mitchell and Gaffan, 2008).

posterior end of the rhinal sulcus. When the lesion was complete, the dura was sewn, the bone flap was replaced and held with sutures, and the skin and galea were closed in layers. Anti-inflammatory (dexamethasone, $1 \mathrm{mg} / \mathrm{kg}$, i.m.), analgesic (buprenorphine, $0.01 \mathrm{mg} / \mathrm{kg}$, i.m.), and antibiotic $(8.75 \mathrm{mg} / \mathrm{kg}$, oral $)$ treatment continued after surgery in consultation with veterinary staff, typically for $5 \mathrm{~d}$.

\section{Histology}

After completion of all behavioral testing, each monkey was sedated with ketamine $(10 \mathrm{mg} / \mathrm{kg})$, deeply anesthetized with intravenous barbiturate, and transcardially perfused with $0.9 \%$ saline followed by $10 \%$ formalin. The brains were cryoprotected in formalin-sucrose, frozen, sectioned coronally $(50 \mu \mathrm{m})$, and stained with cresyl violet. A one-in-five series was collected from the anterior commissure throughout the thalamus for the $\mathrm{MD}+\mathrm{Fx}$ lesions, and a one-in-ten series was collected through the medial temporal lobe for the ERh lesions.

\section{Assessment of lesions}

MDmc lesions. The three monkeys that formed the MD+Fx lesion group had extensive bilateral lesions in the magnocellular division of the mediodorsal thalamic nucleus, in addition to complete transection of the fornix, both as intended (see Figs. 1, 2; supplemental Fig. 1, available at www.jneurosci.org as supplemental material). The extent of the MDmc lesions in the current study was compared with that from a previous study in which $\mathrm{MDmc}$ lesions produced anterograde but not retrograde memory impairments (Mitchell and Gaffan, 2008). This comparative analysis, which measured the area of the individual lesions (Table 1), was performed on an IBM computer using the public domain NIH ImageJ program (developed at the National Institutes of Health and available at http://rsb.info.nih.gov/ij/). In both studies, there was similar additional unavoidable damage to midline thalamic nuclei lying between the MDmc in the two hemispheres of the thalamus. Previous studies have reported that this damage alone does not produce memory impairments (Aggleton and Mishkin, 1983; Gaffan and Murray, 1990). In the current study, all animals also had additional unintended damage to more anterior aspects of the medial thalamus, including left-sided unilateral damage to anterior thalamic nuclei, in particular the anterior medial nucleus (in all three animals) and the anterior ventral nucleus (in two animals: $\mathrm{MD}+\mathrm{Fx} 1$ and $\mathrm{MD}+\mathrm{Fx} 2) . \mathrm{MD}+\mathrm{Fx} 2$ and $\mathrm{MD}+\mathrm{Fx} 3$ also sustained some unintended unilateral left-sided damage to the parvocellular mediodorsal thalamic nucleus. MD + Fx3 sustained slight unintended unilateral left-sided damage to the ventral aspects of the ventral anterior thalamic nucleus. In contrast, only one animal (MD5) from the previous study by Mitchell and Gaffan (2008) sustained additional unintended unilateral damage to anterior thalamus; however, this animal showed no retrograde amnesia. Lesions to the anterior thalamus in macaques produce memory deficits in the within-session new learning version of the object-in-place scene learning task (Parker and Gaffan, 1997). Furthermore, lesions to the anterior thalamus in marmosets and in rats produce deficits in spatial working memory tasks (Ridley et al., 2002, 2004; Mitchell and Dalrymple-Alford, 2006). The object-in-place scene learning task assesses some aspects of spatial working memory (Gaffan, 1994). Thus, this unintended unilateral damage to the anterior thalamus may have contributed, in combination with the bilateral damage to the MDmc and the Fx, to the severity of the cognitive deficits. 
Table 2. Percentage of damage to area ERh in three monkeys with ERh lesions

\begin{tabular}{llll}
\hline Monkey & Left hemisphere & Right hemisphere & Average damage \\
\hline ERh1 & 42.76 & 54.62 & 48.69 \\
ERh2 & 36.35 & 47.91 & 42.13 \\
ERh3 & 45.90 & 54.59 & 50.24 \\
Mean & 41.67 & 52.37 & 47.02 \\
\hline
\end{tabular}

ERh lesions. The three monkeys that formed the ERh lesion group had bilateral ablations to the entorhinal cortex as intended, with very little damage to surrounding medial temporal lobes (see Figs. 3, 4; Table 2). There was very slight damage to the subiculum in the right hemisphere of ERh2 at interaural level +15 and ERh 3 at +12 , respectively. The lesions in all three cases did not extend to the most posterior and lateral aspects of the entorhinal cortex. Thus, our ERh lesions were not complete, yet they were sufficient to produce retention deficits (experiment 1). However, it cannot be ruled out that the remaining intact entorhinal cortex may be critical for new learning or may have been sufficient to allow recovery of function during experiment 2 . For example, monkeys with ERh lesions in the current study demonstrated comparative memory performance to unoperated controls during experiment 2 (relearning) $\sim 1.5$ months after surgery, whereas a previous study has reported transient deficits after entorhinal lesions at least 9 months after surgery (Leonard et al., 1995). Clearly, further studies are needed that assess memory performance with selective, yet more complete, entorhinal cortex lesions.

\section{Statistics}

All data analyses involved initial errors only; that is, no correction trial data were included. The data from experiment 1 (preoperative vs postoperative retrieval) were analyzed using a repeated-measures ANOVA with two within-subject factors (set: A, B, and C; testing phase: preoperative vs postoperative retrieval test) and a between-subject factor of group (MD+Fx vs ERh vs CON), using SPSS software (version 15.0) for Windows (Microsoft). Further designed comparisons of the significant interaction effects were conducted for lesion group by preoperative versus postoperative test. The data from experiment 2 (relearning) were analyzed using a repeated-measures ANOVA with two within-subject factors (repetition: four postoperative cycles of the retrieval test; set: A, B, and $C$ ) and one between-subject factor, lesion group (MD+Fx vs ERh vs $\mathrm{CON})$. Further designed comparisons of the significant interaction of lesion with repetition were performed using the linear component of repetition. For experiment 3 (new learning), the dependent measure, total number of errors, was analyzed using a one-way ANOVA with the between-subject factor of lesion group ( $\mathrm{MD}+\mathrm{Fx}$ vs ERh vs CON). Least significant difference (LSD) post hoc comparisons were conducted to confirm the main effect of lesion group on new learning. Further designed comparisons of the task (preoperative/postoperative retrieval test vs new learning) with lesion were performed using the pooled error term in an ANOVA on the log-transformed data to compare the proportional lesion effects in the two tasks.

\section{Results}

\section{Experiment 1: retrograde amnesia}

Monkeys destined to have bilateral lesions to the MD+Fx or ERh, or to remain as unoperated controls, did not differ in their ability, first, to learn the three sets of 100 scenes (Table 3 ) or second, to remember the scenes as measured by the total number of errors made in the preoperative one-trial retention test (Table 3). In addition, the three groups did not differ in the number of days that intervened between the preoperative one-trial retrieval test and the postoperative one-trial retrieval test [group $\mathrm{MD}+\mathrm{Fx}$, mean $(M)=20$, range $=18-23 \mathrm{~d}$; group $\mathrm{ERh}, M=19.66$, range $=18-22 \mathrm{~d}$; group CON, $M=19$, range $=17-24 \mathrm{~d}$ ]. After surgery, monkeys with lesions to MD+Fx (Figs. 1, 2) or ERh (Figs. 3, 4), unlike the unoperated control monkeys, showed impaired retention of the 300 scene discriminations in the postoperative retrieval test when compared with their performance on the preoperative retrieval test [Figs. 5, 6 (left)]. The two operated groups were impaired postoperatively, relative to controls, and their impairments were approximately similar in severity (Fig. 5). Thus, combined damage to the $\mathrm{MD}+\mathrm{Fx}$ or selective damage to the ERh produces retrograde amnesia. The decrement between preoperative and postoperative tests was similar for the two operated groups (MD+Fx and ERh), which showed a bigger decrement than the control group (Fig. 6, left). Furthermore, the impairments were similar on average in all three of the preoperatively learned sets. ANOVA using a 3 (group: $\mathrm{MD}+\mathrm{Fx}$, ERh, CON) $\times 2$ (testing phase: preoperative, postoperative) $\times 3$ (set: A, B, and C) repeated-measures ANOVA confirmed that there were significant differences for testing phase $\left(F_{(1,2)}=36.33\right.$, $p<0.001)$, and testing phase by lesion group $\left(F_{(2,8)}=5.14, p=\right.$ $0.037)$. Designed comparison of the interaction of testing phase with $\mathrm{MD}+\mathrm{Fx}$ versus $\mathrm{CON}$ using the pooled error term showed a significant effect $\left(t_{(8)}=2.79, p=0.012\right)$. In addition, designed comparison of the interaction of testing phase with ERh versus CON using the pooled error term showed a significant effect $\left(t_{(8)}\right.$ $=2.51, p=0.018$ ).

There were also significant differences for the three sets (Huynh-Feldt, $F_{(2,4)}=19.25, p=0.001$ ) and for the retention levels of the three different sets across testing phase (HuynhFeldt, $\left.F_{(2,8)}=4.43, p=0.029\right)$ that did not vary with lesion. The interaction of set with testing phase reflects that for all three groups, the most recently learned set (set C) showed more forgetting than the other sets between the preoperative and postoperative testing phases (Gaffan, 1993b). There was no evidence of any temporal gradient in retention after any of the lesions. This is in accord with previous studies that have reported that there is no evidence of a temporal gradient after rhinal lesions (Thornton et al., 1997) and after fornix transection (Gaffan, 1993b). Time spent completing the preoperative and postoperative one-trial retrieval tests did not differ for group, testing phase, or set $(F$ values $<1.0$ ).

\section{Experiment 2: relearning}

Table 4 shows individual monkey rates of relearning for each set during three further cycles of the one-trial retrieval test (cycles 2-4) immediately after the initial postoperative one-trial retrieval test. The impairment of groups $\mathrm{MD}+\mathrm{Fx}$ and $\mathrm{ERh}$ were similar in cycle 1 (the one-trial postoperative retrieval test), but in successive cycles of the same repeated test group, ERh showed good relearning, whereas the impairment of group $\mathrm{MD}+\mathrm{Fx}$ stayed constant, relative to the control group (Fig. 7). ANOVA using a 3 (group: $\mathrm{MD}+\mathrm{Fx}, \mathrm{ERh}, \mathrm{CON}) \times 3$ (set: $\mathrm{A}, \mathrm{B}$, and C) $\times 4$ (repetition: four cycles of the retrieval test, including the main postoperative retrieval test) repeated-measures ANOVA showed that there were significant differences for lesion group $\left(F_{(2,8)}=\right.$ 9.33, $p=0.008$ ), repetition of the test (Huynh-Feldt, $F_{(3,24)}=$ $37.51, p=0.001)$, and an interaction of repetition with lesion group (Huynh-Feldt, $F_{(6,24)}=3.34, p=0.028$ ). There was also a significant difference for set (Huynh-Feldt, $F_{(2,4)}=10.72, p=$ $0.01)$ that did not vary by lesion $(F<1.0)$. LSD post hoc comparisons of the lesion group difference confirmed that the $\mathrm{MD}+\mathrm{Fx}$ group made substantially more errors relearning the 300 objectin-place scene discriminations compared with group $\operatorname{CON}(p=$ $0.003)$ and marginally more errors than group ERh $(p=0.057)$. In contrast, relearning rates between group CON and group ERh did not differ $(p=0.105)$.

Furthermore, as Figure 7 indicates, the significant interaction of repetition with lesion group is between group ERh and group $\mathrm{CON}$, with the second repetition of the retrieval test producing 


\begin{tabular}{|c|c|c|c|c|c|c|c|c|c|c|c|c|c|c|c|c|c|}
\hline \multirow[b]{3}{*}{ Case } & \multicolumn{9}{|c|}{ Preop learning } & \multirow{2}{*}{\multicolumn{3}{|c|}{$\begin{array}{l}\text { Preop retrieval test; } \\
\% \text { error }\end{array}$}} & \multirow{2}{*}{\multicolumn{3}{|c|}{$\begin{array}{l}\text { Postop retrieval test; } \\
\% \text { error }\end{array}$}} & \multirow{2}{*}{\multicolumn{2}{|c|}{ Postop new learning }} \\
\hline & \multicolumn{3}{|c|}{ Errors to criterion } & \multicolumn{3}{|c|}{ Sessions to criterion } & \multicolumn{3}{|c|}{$\begin{array}{l}\text { Time until surgery } \\
\text { at criterion }\end{array}$} & & & & & & & & \\
\hline & Set A & Set B & Set C & Set A & Set B & Set C & Set A & Set B & Set C & Set A & Set B & Set C & Set A & Set B & Set C & Set D; errors to criterion & Set D; sessions to criterion \\
\hline CON1 & 343 & 305 & 169 & 14 & 15 & 8 & 49 & 27 & 9 & 28 & 7 & 4 & 21 & 17 & 22 & 291 & 10 \\
\hline CON2 & 710 & 337 & 275 & 30 & 14 & 13 & 45 & 27 & 9 & 22 & 18 & 4 & 20 & 16 & 11 & 205 & 9 \\
\hline CON3 & 573 & 351 & 230 & 22 & 16 & 9 & 49 & 26 & 11 & 12 & 14 & 9 & 12 & 8 & 12 & 164 & 6 \\
\hline CON4 & 987 & 335 & 278 & 42 & 15 & 10 & 54 & 26 & 14 & 15 & 12 & 7 & 24 & 18 & 11 & 204 & 8 \\
\hline CON5 & 815 & 341 & 265 & 33 & 13 & 11 & 48 & 18 & 8 & 22 & 28 & 15 & 25 & 21 & 13 & 272 & 12 \\
\hline Mean & 686.5 & 333.8 & 243.4 & 28.2 & 14.6 & 10.2 & 49.0 & 24.8 & 10.2 & 19.8 & 15.8 & 7.8 & 20.4 & 16 & 13.8 & 227.2 & 9 \\
\hline$M D+F x 1$ & 299 & 170 & 257 & 12 & 8 & 10 & 60 & 39 & 12 & 17 & 18 & 8 & 30 & 24 & 23 & 517 & 23 \\
\hline$M D+F x 2$ & 447 & 211 & 161 & 20 & 9 & 8 & 47 & 26 & 12 & 23 & 19 & 9 & 30 & 14 & 23 & 1021 & 47 \\
\hline$M D+F \times 3$ & 606 & 293 & 224 & 26 & 12 & 10 & 35 & 21 & 8 & 18 & 20 & 11 & 38 & 28 & 25 & 286 & 12 \\
\hline Mean & 450.7 & 224.7 & 214 & 19.33 & 9.67 & 9.33 & 47.33 & 28.67 & 10.67 & 19.33 & 19 & 9.33 & 32.67 & 22 & 23.67 & 608 & 27.33 \\
\hline ERh1 & 155 & 229 & 194 & 7 & 13 & 10 & 47 & 24 & 6 & 25 & 9 & 6 & 27 & 12 & 23 & 179 & 8 \\
\hline ERh2 & 487 & 329 & 274 & 22 & 13 & 15 & 46 & 28 & 8 & 15 & 17 & 6 & 24 & 26 & 14 & 253 & 11 \\
\hline ERh3 & 515 & 358 & 299 & 24 & 14 & 14 & 61 & 38 & 10 & 23 & 18 & 7 & 33 & 30 & 22 & 210 & 8 \\
\hline Mean & 385.7 & 305.3 & 255.7 & 17.67 & 13.33 & 13 & 51.33 & 30 & 8 & 21 & 14.67 & 6.3 & 28 & 22.67 & 19.67 & 214 & 9 \\
\hline
\end{tabular}

The data shown are the total number of presentations of each set to reach the preoperative performance criterion of $>90 \%$ correct across two trials; the elapsed time between reaching criterion for sets $A$, B, and C and surgery; the number of preoperative and postoperative retention errors made during the one-trial retention tests for each set of 100 object-in-place scene discriminations and postoperative new learning of set $D$; the total number of errors made; and number of sessions required to reach criterion of $90 \%$ across two consecutive trials by each monkey. Preop, Preoperative; Postop, postoperative.

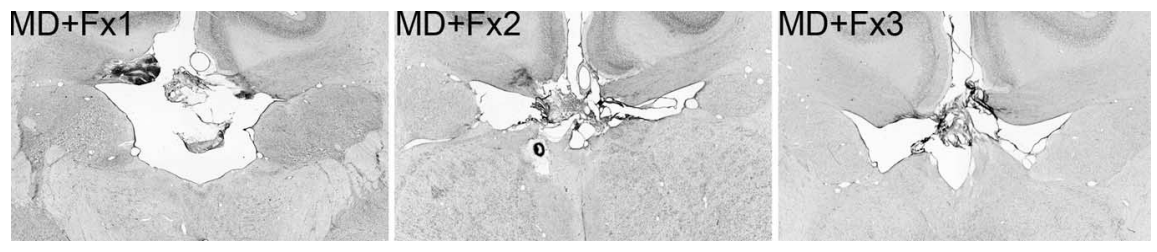

Figure 1. Fornix transection. Photomicrographs of the Fx transection for MD $+F x 1, M D+F x 2$, and MD $+F x 3$ are shown. ment in retrieval errors was similar after the two lesions, whereas in new learning, the proportional increment in learning errors was less than in retrieval for group $\mathrm{ERh}$ and more than in retrieval for group $\mathrm{MD}+\mathrm{Fx}$. To test the specific hypothesis that, proportional to the preoperative scores, the two tasks shown in Figure 6 were differentially sensitive to the effects of the two lesions, MD+Fx and ERh, a de-

more relearning errors because of the poor retention that occurred in the initial postoperative retrieval test (first cycle of the three sets). Designed comparison of the ERh versus CON difference in the linear component of repetitions showed a significant effect $\left(t_{(8)}=2.75, p=0.013\right)$. There was no similar effect in $\mathrm{MD}+\mathrm{Fx}$ versus CON $(t<1.0)$.

\section{Experiment 3: anterograde amnesia}

Despite both lesion groups demonstrating impaired retention of the preoperatively learned scenes in the one-trial retrieval test, only the monkeys with combined bilateral MD+Fx lesions had severe impairments in encoding new information; the ERh lesion group showed similar new learning rates to controls. Group $\mathrm{MD}+\mathrm{Fx}$ was impaired in new postoperative learning, and group ERh was not (Fig. 6, right; for comparison, the errors in preoperative learning of set $\mathrm{C}$ are also presented). Monkeys with $\mathrm{MD}+\mathrm{Fx}$ lesions accumulated more errors to reach the retention criteria of $90 \%$ correct across two consecutive sessions $(M=608$, $\mathrm{SD}=375.9)$ than group $\mathrm{CON}(M=215.8, \mathrm{SD}=40.09)$ and group ERh $(M=233.3, \mathrm{SD}=21.73)$. A one-way ANOVA confirmed that this difference was significant $\left(F_{(2,8)}=4.485, p=\right.$ 0.049). Additional post hoc comparisons confirmed the MD+Fx group made more errors than either group CON $(p=0.022)$ or group ERh $(p=0.042)$, which did not differ. Individual performance data on the acquisition of set D are presented in Table 1.

Furthermore, Figure 6 allows a visual comparison of the results from the postoperative retrieval tests of preoperatively learned sets (left) and from the postoperative new learning of a similar new set (right). It can be seen that, in proportion to the preoperative scores in these two tasks, the proportional incre- signed comparison analyzed the interaction of task and lesion for these two lesions, using the pooled error term in an ANOVA on the log-transformed data to compare the proportional lesion effects in the two tasks. This was a significant contrast $\left(t_{(8)}=2.368\right.$, $p=0.0113$, one-tailed).

\section{Discussion}

The results demonstrate separable memory processes when monkeys are required to retrieve preoperatively acquired information or learn new information after brain damage. In experiment 1 , both $\mathrm{MD}+\mathrm{Fx}$ and ERh ablation caused retrograde amnesia for scene discriminations. This result is unexpected for the MD+Fx lesion group because selective discrete neurotoxic lesions of MDmc alone do not impair retention (Mitchell and Gaffan, 2008), and Fx transection alone produces only very mild retention deficits (Gaffan, 1993a,b; Buckley et al., 2008). Retrograde amnesia after selective ERh lesions has not been reported. The experimental design was ideal for detecting an impairment in retrieval of preoperatively learned information, because all animals showed comparable retention levels preoperatively (Fig. 6, left panel), and the one-trial retrieval test does not allow for any postoperative relearning.

$\mathrm{MD}+\mathrm{Fx}$ impaired both relearning of preoperatively learned scenes as well as new scene learning. The impairment in new scene learning was much greater than the effect of MDmc lesions alone; monkeys with MD+Fx made three times as many errors as monkeys with only selective MDmc lesions in a previously reported study on the same task (Mitchell and Gaffan, 2008). In contrast to the retrograde effects, the ERh lesion group was able 
to relearn the scenes, performing as well as unoperated controls by the third cycle of relearning (Fig. 7), and was unimpaired in new postoperative scene learning.

\section{Retrograde versus anterograde amnesia}

The present data from ERh ablations add to previous evidence that cortical ablations always produce more severe retrograde than anterograde effects. Five experiments, including ours, have combined a one-trial assessment of postoperative retrieval with an assessment of postoperative new learning of the same material after cortical ablations in the temporal lobe. These are by Dean and Weiskrantz (1974), with inferior temporal cortex ablations that spared the rhinal cortex; by Thornton et al. (1997), with combined removals of perirhinal and entorhinal cortex; and by Hampton and Murray (2002) and M. J. Buckley and D. Gaffan (unpublished observation), both with selective perirhinal cortex lesions. The first two experiments assessed object-reward association memory, and the latter two assessed concurrent object discrimination learning; all four experiments found a more severe retrograde than anterograde effect. Within-group comparisons of the magnitude of impairment by Hampton and Murray (2002) (their Figs. 4 and 5) support our notion that the deficits in retention were of a greater magnitude than the deficits in new postoperative learning. Browning and Gaffan (2008), using a similar design, reported a similar finding after crossed unilateral frontal and temporal ablations. Thus, it appears that a general feature of cortical ablations is that they produce a more severe retrograde than anterograde effect when assessed with this one-trial measurement of postoperative retrieval. Many more experiments have looked at postoperative relearning but have not used a sufficient number of discriminations to allow a confident assessment of performance on the first postoperative exposure to each discrimination.

Figure 6 compares anterograde and retrograde effects after the combined $\mathrm{MD}+\mathrm{Fx}$ subcortical lesion. Here, in contrast to the effect of cortical lesions, the anterograde impairment was more severe than the retrograde impairment. Previous experiments have found that both MDmc lesions alone and Fx transection alone produce a more severe anterograde than retrograde effect (Gaffan, 1993a,b; Mitchell and Gaffan, 2008). Thus, both cortical removals and subcortical lesions can produce both anterograde and retrograde deficits, their overall severity increasing with lesion extent, but cortical ablations produce more severe retrograde than anterograde ef-

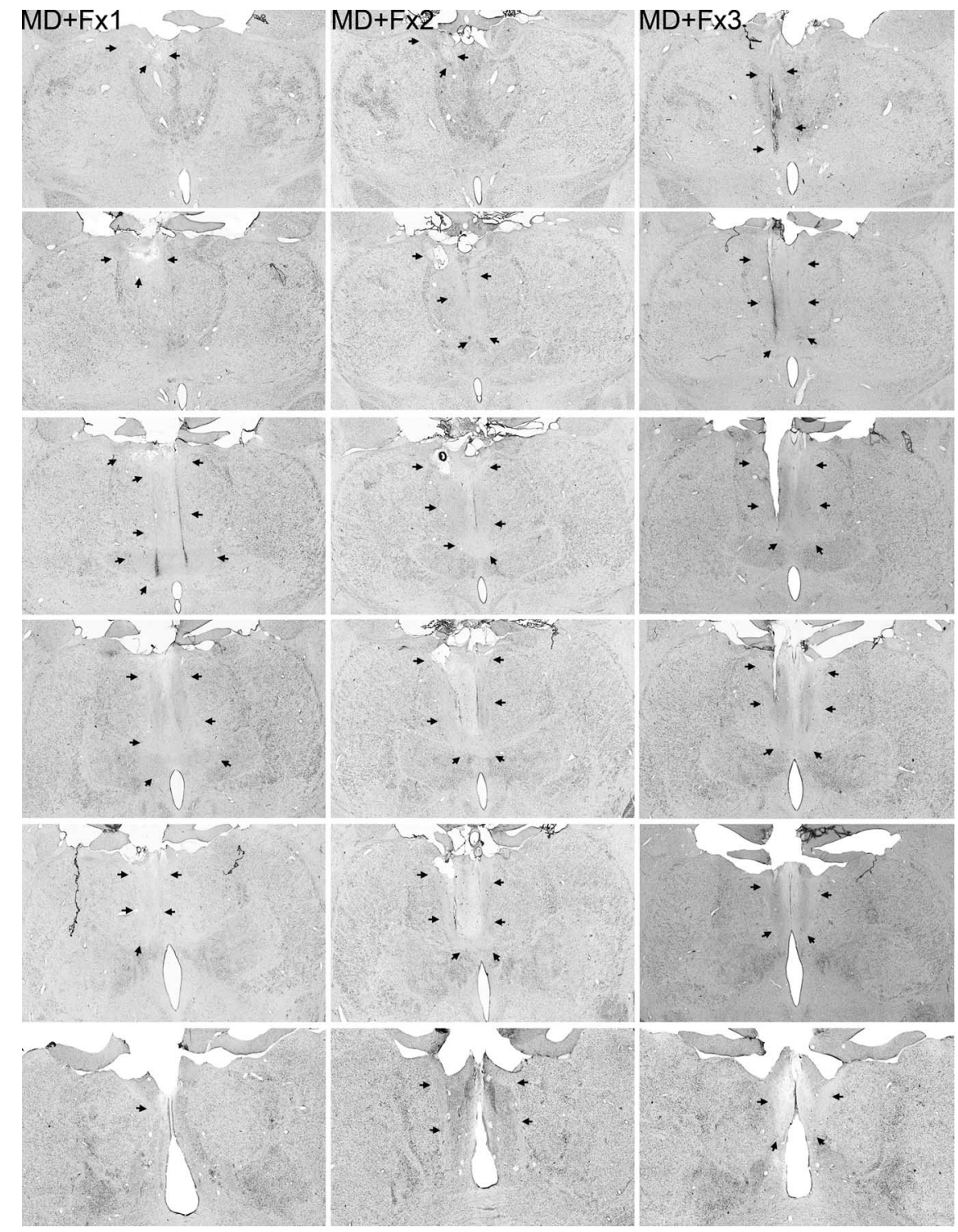

Figure 2. Magnocellular mediodorsal thalamus lesions. Photomicrographs of the MDmc lesions for MD $+F x 1, M D+F \times 2$, and $\mathrm{MD}+\mathrm{F} \times 3$ are shown, corresponding as closely as possible to NORM (normal medial thalamus) and the schematic drawings of supplemental Fig. 1, available at www.jneurosci.org as supplemental material. Arrows indicate the borders of each lesion.
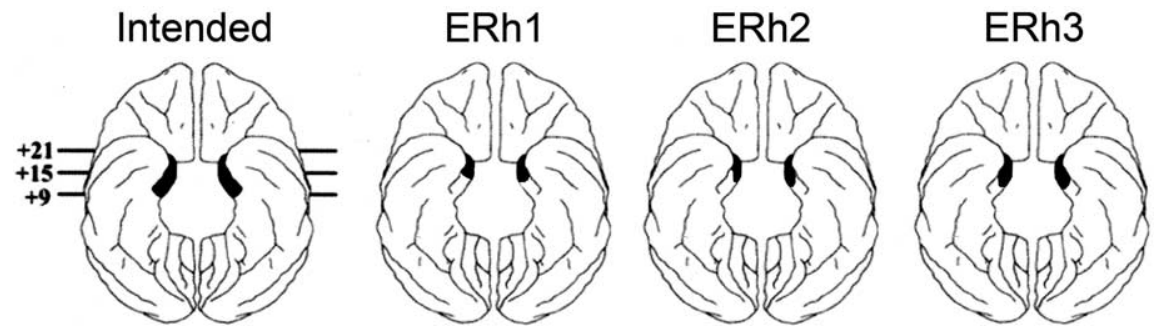

Figure 3. Ventral view of the entorhinal ablations in the macaque brain. The intended location and extent of the lesion to the entorhinal cortex is illustrated by the shaded region on the schematic diagram on the far left. The shaded regions on the other three diagrams represent the actual extent of the ablations to animals ERh1, ERh2, and Erh3. The numerals represent the distance in millimeters from the interaural plane. fects, whereas the retrograde effects of subcortical lesions are less severe than their anterograde effects. It must be reiterated that we do not claim that selective cortical ablations do not severely impair new postoperative learning in certain conditions (Buckley 

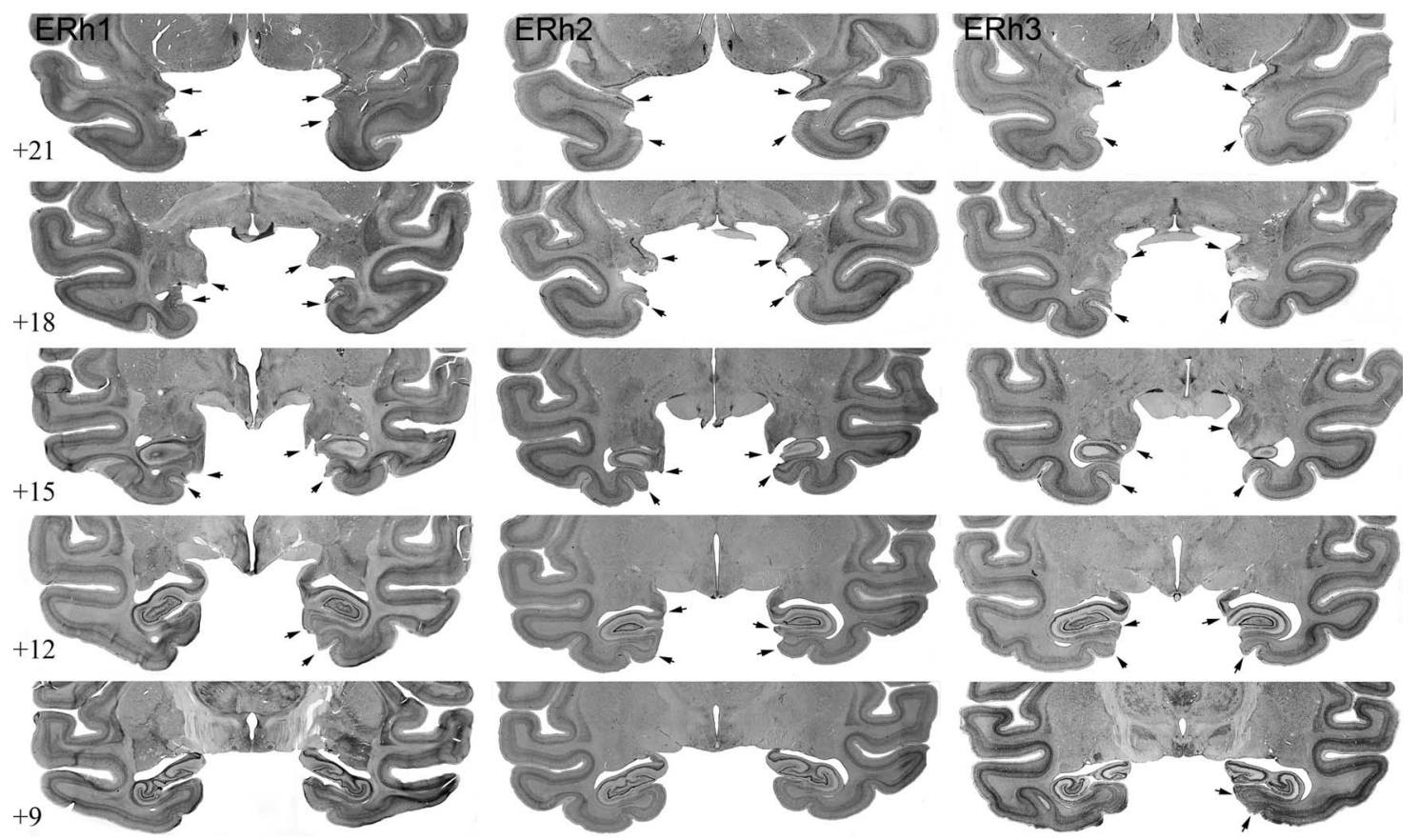

Figure 4. Entorhinal ablations. Photomicrographs of the ERh lesions for ERh1, ERh2, and ERh3 are shown. Each row represents one approximate stereotaxic level, in millimeters anterior to the interaural plane, from anterior to posterior. The arrows indicate the extent of the lesion in each animal.

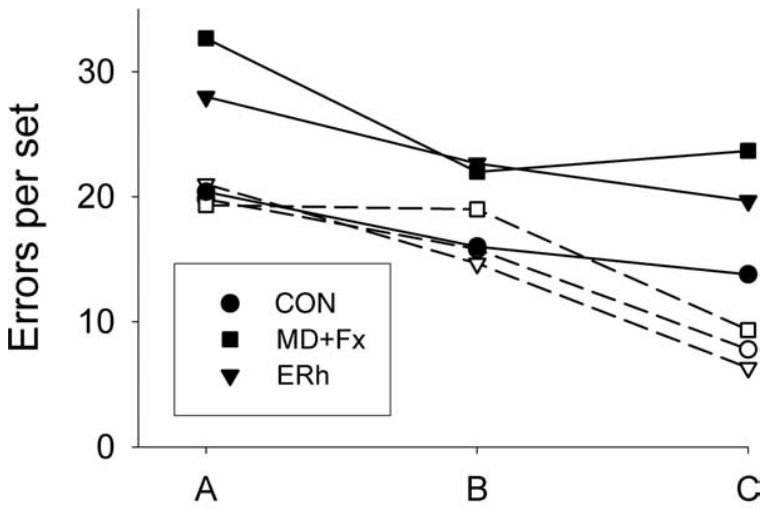

Pre-operatively learned set

Figure 5. Preoperative and postoperative retrieval tests. The total mean errors per set for each group (CON, MD + Fx, and ERh) is shown for each of the three preoperatively learned sets. Open symbols, Preoperative one-trial retrieval test; filled symbols, postoperative one-trial retrieval test.

and Gaffan, 1998; Buckley et al., 2001; Bussey et al., 2002, 2003). Rather, based on the results from the current study, we propose that compared with retention deficits, new learning deficits are milder after selective cortical ablations, and the converse is true for subcortical lesions.

\section{Subcortical structures and retention}

Bright et al. (2006) have proposed that a widely distributed network of regions extending beyond the medial temporal lobe underlies the retrieval of past memories. Our evidence demonstrating severe and stable retrograde amnesia after $\mathrm{MD}+\mathrm{Fx}$ may support this proposal. We propose that the retention deficit caused by $\mathrm{MD}+\mathrm{Fx}$ is caused by disruptions in widely distributed neural networks via their interconnections with the prefrontal cortex and medial temporal lobes. The MDmc has strong recip- rocal connections with prefrontal cortex, especially the orbitofrontal region (Goldman-Rakic and Porrino, 1985; Russchen et al., 1987; Behrens et al., 2003), and also receives input from perirhinal cortex and the amygdala (Aggleton and Mishkin, 1984; Russchen et al., 1987; Saunders et al., 2005). The Fx interconnects the extended hippocampal system, including the medial temporal lobes, with the medial diencephalon and other subcortical structures (Swanson, 1977; Aggleton and Saunders, 1997; Saunders and Aggleton, 2007). Because discrete selective lesions to MDmc or Fx alone do not cause severe retrograde impairments (Gaffan, 1993a,b; Mitchell and Gaffan, 2008), our current finding suggests that for retrograde amnesia to occur as a result of subcortical lesions, the damage must be extensive. That is, combined MDmc and Fx lesions disconnect and disrupt enough of the widespread, distributed cortical network (Bright et al., 2006) that retrieval is impaired. This may help to explain dense amnesia in humans (Kopelman et al., 2003) after subcortical damage, because damage in these cases is rarely limited to a single subcortical structure.

\section{Entorhinal cortex and new learning}

The lack of any postoperative learning impairment for the ERh group was unexpected because a previous study reported impaired within-session learning of new object-in-place scene discriminations after ERh lesions (Charles et al., 2004). The ERh lesions in that study also produced damage to perirhinal cortex which probably accounts for this difference, although their monkey labeled "Ent D" had a selective, smaller ERh lesion that produced a relatively small deficit in new learning. Possibly, rapid learning of scenes within daily test sessions is more sensitive to a small impairment in new learning caused by ERh damage. Alternatively, ERh lesions impair demanding visual memory tasks requiring flexible manipulation of memory for stimulus relationships (Buckmaster et al., 2004) but not two-choice object discrimination learning (Thornton et al., 1997). Because objectin-place scene-discrimination learning does not explicitly require 

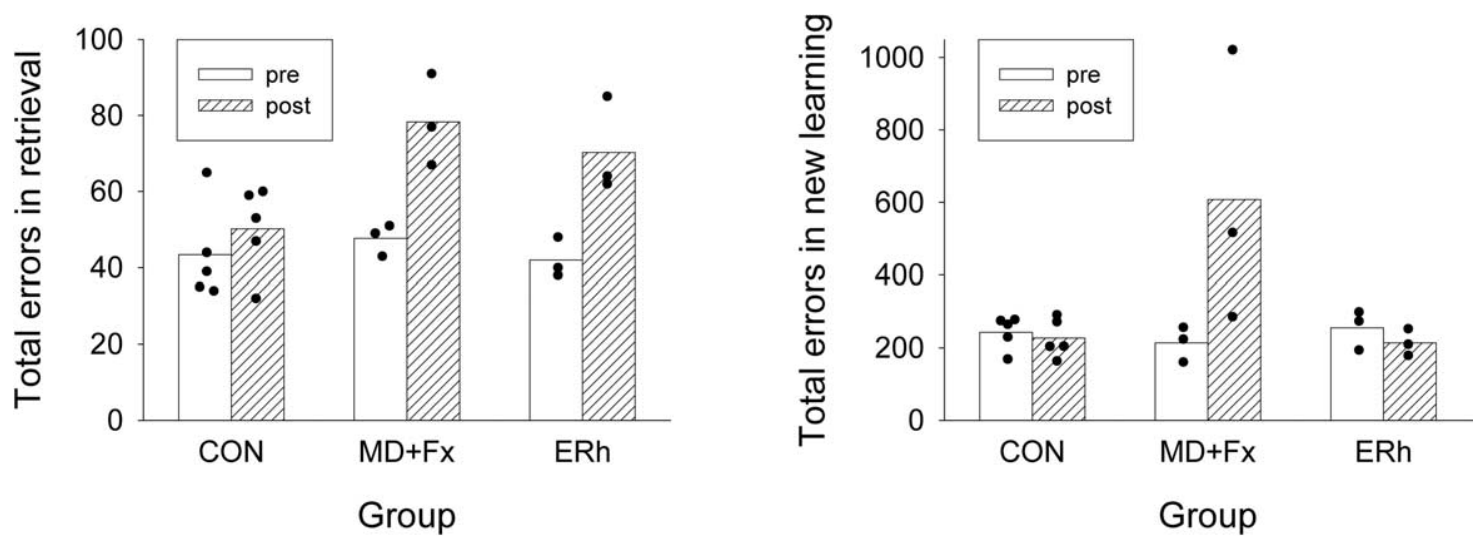

Figure 6. Preoperative and postoperative tests. Left, Total errors in retrieval, for individual subjects and for group means (CON, MD $+F x$, and ERh), during preoperative (pre) and postoperative (post) performance in one-trial retrieval tests, summed over all three of the preoperatively learned sets (A, B, and C). Right, Total errors in new learning of 100 novel, unique scene discriminations to $90 \%$ correct across two consecutive testing sessions, for individual subjects and for group means, during the final preoperatively learned set (set $\mathrm{C}$ ) and during the postoperatively learned novel set (set D).

Table 4. Postoperative relearning

\begin{tabular}{|c|c|c|c|c|c|c|c|c|c|}
\hline \multirow[b]{2}{*}{ Case } & \multicolumn{3}{|c|}{ Cycle 2} & \multicolumn{3}{|c|}{ Cycle 3} & \multicolumn{3}{|c|}{ Cycle 4} \\
\hline & Set A & Set B & Set C & Set A & Set B & Set C & Set A & Set B & Set C \\
\hline CON1 & 17 & 9 & 15 & 12 & 10 & 19 & 11 & 9 & 13 \\
\hline CON2 & 12 & 13 & 10 & 14 & 10 & 7 & 13 & 10 & 10 \\
\hline $\mathrm{CON} 3$ & 15 & 10 & 8 & 17 & 7 & 6 & 10 & 4 & 5 \\
\hline CON4 & 15 & 16 & 13 & 24 & 14 & 10 & 18 & 6 & 7 \\
\hline CON5 & 19 & 14 & 18 & 21 & 16 & 8 & 14 & 15 & 11 \\
\hline Mean & 15.6 & 12.4 & 12.8 & 17.6 & 11.4 & 10 & 13.2 & 8.8 & 9.2 \\
\hline$M D+F \times 1$ & 23 & 26 & 17 & 23 & 21 & 22 & 22 & 22 & 18 \\
\hline$M D+F \times 2$ & 27 & 21 & 16 & 20 & 22 & 22 & 19 & 15 & 21 \\
\hline$M D+F \times 3$ & 21 & 26 & 18 & 21 & 21 & 16 & 12 & 18 & 14 \\
\hline Mean & 23.6 & 24.33 & 17 & 21.33 & 21.33 & 20 & 17.67 & 18.33 & 17.67 \\
\hline ERh1 & 26 & 8 & 12 & 15 & 5 & 6 & 8 & 9 & 4 \\
\hline ERh2 & 32 & 23 & 12 & 25 & 13 & 11 & 14 & 15 & 10 \\
\hline ERh3 & 23 & 25 & 22 & 13 & 15 & 17 & 11 & 13 & 11 \\
\hline Mean & 27 & 18.67 & 15.33 & 17.67 & 11 & 11.33 & 11 & 12.33 & 8.33 \\
\hline
\end{tabular}

Postoperative relearning rates of each set during three further repetitions (Cycles 2-4) of the one-trial retrieval test immediately following the initial postoperative one-trial retrieval test.

flexible use of memory, the involvement of ERh in this task may be limited. Notwithstanding these considerations, the present evidence from ERh ablations adds to previous evidence that cortical ablations always produce more severe retrograde than anterograde effects.

\section{Subcortical structures and new learning}

Several studies have highlighted the activation of the thalamus during encoding of visual information. Recent functional magnetic resonance imaging evidence shows significant activation of thalamus during encoding of information and immediate retrieval but no thalamic activation during delayed retrieval (Neuner et al., 2007). In accord with this, electrophysiological evidence shows that as a stimulus is no longer novel, activation in the MDmc diminishes (Fahy et al., 1993). It has been proposed that this activation may signal the role of the thalamus in attention associated with encoding. Consistent with this view, patients with thalamic damage also have executive function and attention deficits (Van der Werf et al., 2003). Others have suggested that the role of the thalamus may be to pass on combinations of associations from the medial temporal lobes to the cortex. For example, the $\mathrm{MDmc}$ receives inputs from perirhinal cortex, area TE (anterior inferotemporal cortex) and amygdala (Russchen et al., 1987), whereas the anterior thalamus receives inputs via the fornix from the extended hippocampal system and mammillary bodies. These respective inputs may then combine within thalamus and influence prefrontal cortex and cingulate cortex functioning, respectively (Aggleton and Brown, 1999). Our current study challenges the emphasis placed on identifying one critical region responsible for dense amnesia and other cognitive processes associated with the medial diencephalon. Instead, our findings extend the proposal that dense amnesias associated with the medial diencephalon are the result of widespread subcortical disconnection causing both retrograde and anterograde amnesia. Aggleton and Brown (1999) proposed that an extended hippocampal circuit including the anterior thalamus, mammillary bodies, and fornix is responsible for episodic memory processing especially in relation to object-in-place information. We propose that MDmc and its respective interconnections are also important for processing information, in parallel, about object, reward, and value, attributes that are also critical for episodic memory and new learning in general (Mitchell and Dalrymple-Alford, 2005; Mitchell et al., 2007a,b; Mitchell and Gaffan, 2008).

The present data provide direct evidence that retention, relearning, and encoding are subserved by independent neural systems in the brain. We conclude that damage to subcortical structures must be widespread in order for the consequent disruption of cortical processing to cause profound retrograde amnesia. Fur- 


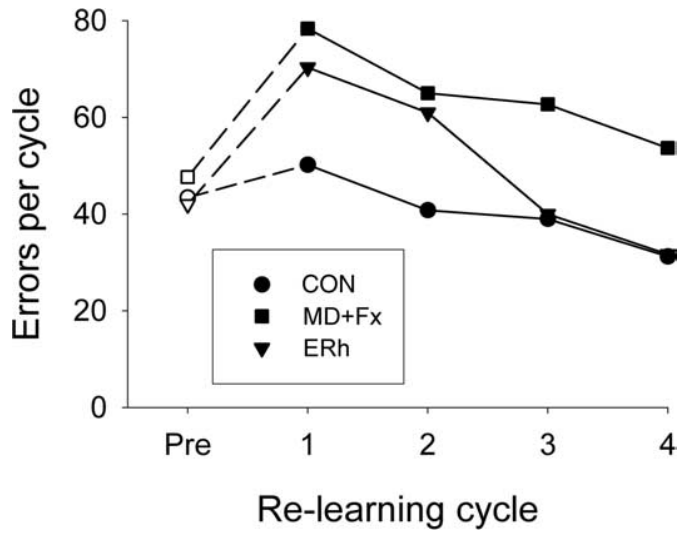

Figure 7. Relearning. The total mean errors summed over all three of the preoperatively learned sets ( $A, B$, and $C$ ) for each group (CON, MD + Fx, and ERh). The relearning cycles Pre and 1 are the preoperative and postoperative one-trial retrieval tests, respectively; cycles 2,3, and 4 are further repeats of the same test. Open symbols, Preoperative one-trial retrieval test; filled symbols, postoperative one-trial retrieval test.

thermore, removal of cortical neurons produces a greater impairment in memory retrieval than in new learning, whereas subcortical damage produces a greater impairment in new learning than in memory retrieval. These conclusions challenge traditional views that only the medial temporal lobes are important for memory processing (Squire et al., 2004). Our current study also challenges the emphasis placed on identifying one critical region responsible for dense amnesia and other cognitive processes associated with the medial diencephalon. They also suggest that damage to more than one single critical structure, pathway, or neuromodulatory influence is responsible for dense amnesia.

\section{References}

Aggleton JP, Brown MW (1999) Episodic memory, amnesia, and the hippocampal-anterior thalamic axis. Behav Brain Sci 22:425-444; discussion 444-489.

Aggleton JP, Brown MW (2006) Interleaving brain systems for episodic and recognition memory. Trends Cogn Sci 10:455-463.

Aggleton JP, Mishkin M (1983) Visual recognition impairment following medial thalamic lesions in monkeys. Neuropsychologia 21:189-197.

Aggleton JP, Mishkin M (1984) Projections of the amygdala to the thalamus in the cynomolgus monkey. J Comp Neurol 222:56-68.

Aggleton JP, Saunders RC (1997) The relationships between temporal lobe and diencephalic structures implicated in anterograde amnesia. Memory 5:49-71.

Behrens TE, Johansen-Berg H, Woolrich MW, Smith SM, Wheeler-Kingshott CA, Boulby PA, Barker GJ, Sillery EL, Sheehan K, Ciccarelli O, Thompson AJ, Brady JM, Matthews PM (2003) Non-invasive mapping of connections between human thalamus and cortex using diffusion imaging. Nat Neurosci 6:750-757.

Bright P, Buckman J, Fradera A, Yoshimasu H, Colchester AC, Kopelman MD (2006) Retrograde amnesia in patients with hippocampal, medial temporal, temporal lobe, or frontal pathology. Learn Mem 13:545-557.

Browning PG, Gaffan D (2008) Global retrograde amnesia but selective anterograde amnesia after frontal-temporal disconnection in monkeys. Neuropsychologia 46:2494-2502.

Buckley MJ, Gaffan D (1998) Perirhinal cortex ablation impairs visual object identification. J Neurosci 18:2268-2275.

Buckley MJ, Booth MC, Rolls ET, Gaffan D (2001) Selective perceptual impairments after perirhinal cortex ablation. J Neurosci 21:9824-9836.

Buckley MJ, Wilson CR, Gaffan D (2008) Fornix transection impairs visuospatial memory acquisition more than retrieval. Behav Neurosci 122:44-53.

Buckmaster CA, Eichenbaum H, Amaral DG, Suzuki WA, Rapp PR (2004) Entorhinal cortex lesions disrupt the relational organization of memory in monkeys. J Neurosci 24:9811-9825.
Bussey TJ, Saksida LM, Murray EA (2002) Perirhinal cortex resolves feature ambiguity in complex visual discriminations. Eur J Neurosci 15:365-374.

Bussey TJ, Saksida LM, Murray EA (2003) Impairments in visual discrimination after perirhinal cortex lesions: testing 'declarative' vs. 'perceptualmnemonic' views of perirhinal cortex function. Eur J Neurosci 17:649-660.

Charles DP, Browning PG, Gaffan D (2004) Entorhinal cortex contributes to object-in-place scene memory. Eur J Neurosci 20:3157-3164.

Dean P, Weiskrantz L (1974) Loss of preoperative habits in Rhesus monkeys with inferotemporal lesions: recognition failure or relearning deficit? Neuropsychologia 12:299-311.

Fahy FL, Riches IP, Brown MW (1993) Neuronal signals of importance to the performance of visual recognition memory tasks: evidence from recordings of single neurones in the medial thalamus of primates. Prog Brain Res 95:401-416.

Gaffan D (1993a) Normal forgetting, impaired acquisition in memory for complex naturalistic scenes by fornix-transected monkeys. Neuropsychologia 31:403-406.

Gaffan D (1993b) Additive effects of forgetting and fornix transection in the temporal gradient of retrograde-amnesia. Neuropsychologia 31:1055-1066.

Gaffan D (1994) Scene-specific memory for objects: a model of episodic memory impairment in monkeys with fornix transection. J Cogn Neurosci 6:305-320.

Gaffan D, Murray EA (1990) Amygdalar interaction with the mediodorsal nucleus of the thalamus and the ventromedial prefrontal cortex in stimulus reward associative learning in the monkey. J Neurosci 10:3479-3493.

Goldman-Rakic PS, Porrino LJ (1985) The primate mediodorsal (MD) nucleus and its projection to the frontal lobe. J Comp Neurol 242:535-560.

Graff-Radford NR, Tranel D, Van Hoesen GW, Brandt JP (1990) Diencephalic amnesia. Brain 113:1-25.

Hampton RR, Murray EA (2002) Learning of discriminations is impaired, but generalization to altered views is intact, in monkeys (Macaca mulatta) with perirhinal cortex removal. Behav Neurosci 116:363-377.

Hodges JR, McCarthy RA (1993) Autobiographical amnesia resulting from bilateral paramedian thalamic infarction. A case study in cognitive neurobiology. Brain 116:921-940.

Ilinsky IA, Kultas-Ilinsky K (1987) Sagittal cytoarchitectonic maps of the Macaca mulatta thalamus with a revised nomenclature of the motorrelated nuclei validated by observations on their connectivity. J Comp Neurol 262:331-364.

Kopelman MD (2002) Disorders of memory. Brain 125:2152-2190.

Kopelman MD, Stanhope N, Kingsley D (1999) Retrograde amnesia in patients with diencephalic, temporal lobe or frontal lesions. Neuropsychologia 37:939-958.

Kopelman MD, Lasserson D, Kingsley DR, Bello F, Rush C, Stanhope N, Stevens TG, Goodman G, Buckman JR, Heilpern G, Kendall BE, Colchester AC (2003) Retrograde amnesia and the volume of critical brain structures. Hippocampus 13:879-891.

Leonard BW, Amaral DG, Squire LR, Zola-Morgan S (1995) Transient memory impairment in monkeys with bilateral lesions of the entorhinal cortex. J Neurosci 15:5637-5659.

Meunier M, Hadfield W, Bachevalier J, Murray EA (1996) Effects of rhinal cortex lesions combined with hippocampectomy on visual recognition memory in rhesus monkeys. J Neurophysiol 75:1190-1205.

Miller LA, Caine D, Watson JD (2003) A role for the thalamus in memory for unique entities. Neurocase 9:504-514.

Mitchell AS, Dalrymple-Alford JC (2005) Dissociable memory effects after medial thalamus lesions in the rat. Eur J Neurosci 22:973-985.

Mitchell AS, Dalrymple-Alford JC (2006) Lateral and anterior thalamic lesions impair independent memory systems. Learn Mem 13:388-396.

Mitchell AS, Gaffan D (2008) The magnocellular mediodorsal thalamus is necessary for memory acquisition, but not retrieval. J Neurosci 28:258-263.

Mitchell AS, Browning PG, Baxter MG (2007a) Neurotoxic lesions of the medial mediodorsal nucleus of the thalamus disrupt reinforcer devaluation effects in rhesus monkeys. J Neurosci 27:11289-11295.

Mitchell AS, Baxter MG, Gaffan D (2007b) Dissociable performance on scene memory learning and strategy implementation after lesions to magnocellular mediodorsal thalamic nucleus. J Neurosci 27:11888-11895.

Neuner I, Stöcker T, Kellermann T, Kircher T, Zilles K, Schneider F, Shah NJ 
(2007) Wechsler Memory Scale Revised Edition: neural correlates of the visual paired associates subtest adapted for fMRI. Brain Res 1177:66-78.

Parker A, Gaffan D (1997) The effect of anterior thalamic and cingulate cortex lesions on object-in-place memory in monkeys. Neuropsychologia 35:1093-1102.

Ridley RM, Maclean CJ, Young FM, Baker HF (2002) Learning impairments in monkeys with combined but not separate excitotoxic lesions of the anterior and mediodorsal thalamic nuclei. Brain Res 950:39-51.

Ridley RM, Baker HF, Mills DA, Green ME, Cummings RM (2004) Topographical memory impairments after unilateral lesions of the anterior thalamus and contralateral inferotemporal cortex. Neuropsychologia 42:1178-1191.

Russchen FT, Amaral DG, Price JL (1987) The afferent input to the magnocellular division of the mediodorsal thalamic nucleus in the monkey, Macaca fascicularis. J Comp Neurol 256:175-210.

Saunders RC, Aggleton JP (2007) Origin and topography of fibers contributing to the fornix in macaque monkeys. Hippocampus $17: 396-411$
Saunders RC, Mishkin M, Aggleton JP (2005) Projections from the entorhinal cortex, perirhinal cortex, presubiculum, and parasubiculum to the medial thalamus in macaque monkeys: identifying different pathways using disconnection techniques. Exp Brain Res 167:1-16.

Squire LR, Zola-Morgan S (1991) The medial temporal lobe memory system. Science 253:1380-1386

Squire LR, Stark CE, Clark RE (2004) The medial temporal lobe. Annu Rev Neurosci 27:279-306.

Swanson LW (1977) The anatomical organization of septo-hippocampal projections. Ciba Found Symp 58:25-48.

Thornton JA, Rothblat LA, Murray EA (1997) Rhinal cortex removal produces amnesia for preoperatively learned discrimination problems but fails to disrupt postoperative acquisition and retention in rhesus monkeys. J Neurosci 17:8536-8549.

Van der Werf YD, Scheltens P, Lindeboom J, Witter MP, Uylings HB, Jolles J (2003) Deficits of memory, executive functioning and attention following infarction in the thalamus; a study of 22 cases with localised lesions. Neuropsychologia 41:1330-1344. 